\title{
Descrição lexical histórico-comparativa de mudanças fonológicas dos sinais da língua francesa para as línguas americana e brasileira de sinais
}

\author{
Historical-comparative lexical description of phonological changes in French to \\ American and Brazilian sign languages
}

\author{
Walber Gonçalves de ABREU* \\ Universidade Federal Rural da Amazônia (UFRA) \\ Carlene Ferreira Nunes SALVADOR ** \\ Universidade Federal Rural da Amazônia (UFRA)
}

\begin{abstract}
RESUMO: O interesse dos franceses em educar os surdos possibilitou que a língua de sinais francesa - LSF se desenvolvesse e influenciasse o surgimento de outras línguas de sinais como a Americana - ASL e a Brasileira - Libras, estabelecendo, portanto, um grau de parentesco entre essas línguas. Diante disso, o objetivo deste trabalho é descrever as mudanças ocorridas da LSF para a ASL e a Libras e demonstrar quais os processos fonológicos envolvidos nessas mudanças. Selecionamos 21 sinais, 7 de cada língua de sinais que identificamos terem semelhanças paramétricas. Após seleção e organização dos dados, analisamos esses sinais qualitativamente e então, identificamos tendências gerais de mudanças fonológicas. Concluímos que os sinais da ASL sofreram mudanças mais consideráveis do que da Libras e que na ASL houve uma tendência em deslocar os sinais que eram realizados na locação boca, na Libras isso ocorreu apenas em 2 sinais, os demais sofreram apenas algumas mudanças simples em sua constituição paramétrica. A partir dos dados, identificamos três tendências gerais de mudanças.
\end{abstract}

PALAVRAS-CHAVE: Linguística Histórica. Línguas de Sinais. LSF. ASL. Libras.

\begin{abstract}
The French interest in educating the deaf enabled the French sign language - LSF to develop and influence the emergence of other sign languages such as American - ASL and Brazilian - Libras, thus establishing a degree of kinship between these languages. Therefore, the objective of this paper is to describe the changes that occurred from LSF to ASL and Libras and to demonstrate which phonological processes are involved in these changes. We selected 21

\footnotetext{
* Mestre em Letras - Estudos Linguísticos do Programa de Pós-Graduação em Letras (PPGL/UFPA). Docente da Universidade Federal Rural da Amazônia (UFRA). E-mail: walber.abreu @ hotmail.com.

${ }^{* *}$ Doutora em Letras - Estudos Linguísticos do Programa de Pós-Graduação em Letras (PPGL/UFPA). Docente da Universidade Federal Rural da Amazônia (UFRA). E-mail: carlene.salvador77@gmail.com.
}

Revista Moara, n. 55, jan-jul 2020 ISSN: 0104-0944

Recebido em 25/03/2020

Avaliado em 25/06/2020 
signs, 7 from each sign language that we identified to have parametric similarities. After selecting and organizing the data, we analyzed these signs qualitatively and then identified general trends in phonological changes. We concluded that the signs of ASL underwent more considerable changes than Libras and that in ASL there was a tendency to displace the signs that were made in the mouth location, in Libras this occurred only in 2 signs, the others suffered only a few simple changes in their parametric constitution. From the data, we identified three general trends for change.

KEYWORDS: Historical Linguistics. Sign Languages. LSF. ASL. Libras.

\section{Introdução}

A história das línguas de sinais mostra que há relação de parentesco entre as línguas de sinais francesa - LSF, americana - ASL e brasileira - Libras. Pelo fato de os franceses terem demonstrado, inicialmente, mais interesse em educar os surdos, essa língua se desenvolveu e influenciou o surgimento de outras que compartilham com ela semelhanças linguísticas.

Nesse sentido, percebemos que alguns sinais da ASL e da Libras derivaram da LSF e a partir do uso em seus respectivos países, os sinais derivados sofreram algumas mudanças. Dessa maneira, para nortear esta pesquisa, foram elencados os seguintes questionamentos: diacronicamente, quais foram as mudanças linguísticas que ocorreram nos sinais da LSF para a ASL e a Libras? E, quais processos fonológicos evidenciam essas mudanças?

Com isso, o objetivo deste trabalho consiste em efetuar a descrição lexical das mudanças ocorridas da LSF para a ASL e a Libras e demonstrar quais processos fonológicos subjazem nessas mudanças. Para isso, foi realizada coleta de sinais de três dicionários: Iconographie des signes, Handspeak e Dicionário da Língua de Sinais do Brasil e desses, foram escolhidos sinais os quais evidenciamos terem semelhanças paramétricas.

Para efeito de sistematização, o trabalho está organizado da seguinte forma: apresentamos questões relativas à linguística histórica e às línguas de sinais em questão. Em seguida, tratamos sobre a metodologia escolhida para o desenvolvimento da 
pesquisa. Após, demonstramos os dados e suas respectivas análises. Finalizamos o trabalho tecendo nossas considerações finais.

\section{Pressupostos teóricos}

\subsection{Linguística Histórica e as Línguas de Sinais}

A história desse campo de estudo mostra que os pesquisadores dessa área começaram a voltar sua atenção para a aparente semelhança entre línguas distintas, no sentido proposto por Gabas Junior (2012, p. 85), “[...] a Linguística Histórica estuda os processos de mudança das línguas". O juiz Sir William Jones, no século XVIII, percebeu relações de parentescos entre o latim, o grego e o sânscrito, mais tarde Jacob Grimm e Karl Verner desenvolveram leis gerais de mudanças de sons entre diferentes línguas, que ficaram conhecidas como 'Leis de Grimm' e Leis de Verner' (GABAS JR, 2012).

Paixão de Sousa (2006) afirma que em 1800 os pesquisadores aliaram a esfera documental com a esfera experimental criando uma abordagem dentro da Linguística Histórica, a abordagem histórico-comparada. Essa abordagem pesquisava as semelhanças entre os idiomas, o que levaria a proposição de uma identidade comum (língua mãe) e ao mesmo tempo evidenciasse as diferenças entre essas línguas, o que os levariam a explicar as mudanças, "entretanto, nos diferentes processos de diversificação, a tradição histórico-comparada observará regularidades compatíveis com a ideia de herança comum" (PAIXÃO DE SOUSA, 2006, p. 15)

Diante disso, o histórico de difusão das línguas de sinais pelo mundo nos faz chegar a graus de parentesco entre línguas de sinais que são comprovados por semelhanças linguísticas. Historicamente, Rocha (2008) relata que em 1815 o reverendo Thomas Hopkins Gallaudet foi até a cidade Paris realizar estudos no instituto de Surdos com o então diretor abade Sicard, depois retornou aos Estados Unidos e juntamente com o professor surdo Laurent Clérc criaram a primeira escola de surdos da América, hoje conhecida como Universidade Gallaudet.

No Brasil, em 1855, o professor surdo francês E. Huet oriundo do Instituto de Surdos de Paris veio até o país e apresentou um relatório a D. Pedro II com o objetivo de criar uma escola para surdos, tendo sido a proposta aceita pelo imperador. 
Atualmente, o instituto idealizado por Huet é conhecido como Instituto Nacional de Educação de Surdos - INES (ROCHA, 2008). Essas evidências nos demonstra a influência da LSF no surgimento da ASL e da Libras.

Deste modo, podemos criar o seguinte esquema arbóreo de relações entre essas três línguas de sinais (Figura 1).

Figura 1 - Esquema arbóreo do tronco LSF

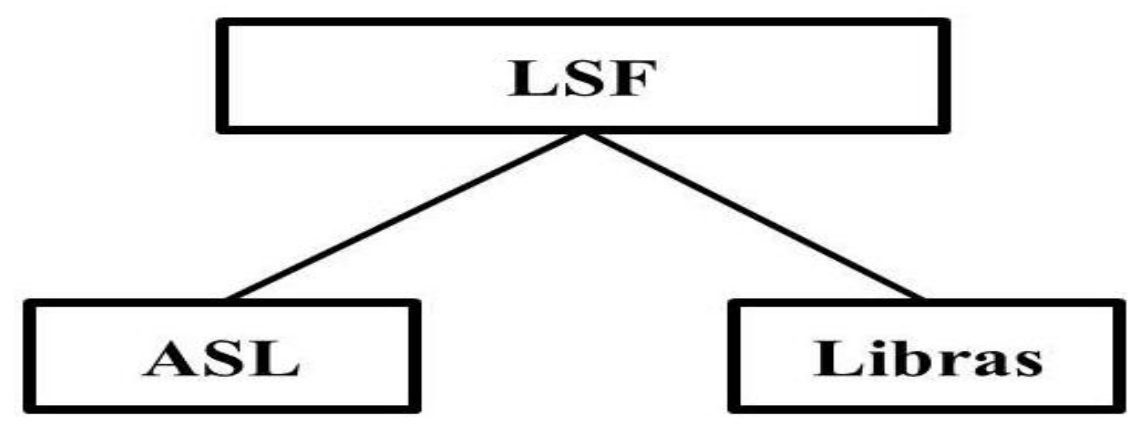

Fonte: Elaboração dos autores, 2020

A Figura 1 ilustra a relação de filiação da ASL e da Libras em relação à LSF. Essa filiação é basicamente oriunda do pioneirismo francês e do tratamento direcionado ao indivíduo surdo nas diferentes esferas da vida cotidiana, inclusive a vida escolar.

\subsection{Processos Fonológicos de Mudança nas Línguas de Sinais}

Busca-se, neste tópico, mostrar os processos fonológicos de mudança nas Línguas de Sinais com vistas a descrever como alguns sinais, a partir de novos contextos, podem assumir formas diferentes.

Diniz (2010 apud FRISHBERG, 1975), apresenta o processo fonológico de mudança paramétrica quanto a configuração de mãos (CM), movimento (MOV), locação (LOC) e orientação da palma da mão (OP) ${ }^{1}$. A partir desses processos primários são apresentadas tendências de mudança nas línguas de sinais, são eles: (a) simetria de duas mãos, (b) deslocamento locativo, (c) conteúdo lexical para as mãos, (d) assimilação e (e) deletação de uma mão. Além disso, os sinais podem permanecer com as mesmas configurações paramétricas, são os chamados sinais idênticos.

\footnotetext{
${ }^{1} \mathrm{CM}$ é a forma da mão na realização do sinal, MOV é o movimento que essa configuração vai realizar no corpo ou no espaço neutro, LOC é onde essa CM se localizará e OP é a direção na qual a palma da mão indicará (QUADROS; KARNOPP, 2004).
}

Revista Moara, n. 55, jan-jul 2020 ISSN: 0104-0944

Recebido em 25/03/2020

Avaliado em 25/06/2020 
Grosso modo, apresenta-se a seguir a configuração de cada tendência:

(a) Simetria de duas mãos pode ocorrer de duas formas. Nos sinais que utilizam duas mãos com CM distintas, a mão passiva $^{2}$ tende a copiar a $\mathrm{CM}$ e o MOV da mão ativa $^{3}$. Nos sinais que utilizam apenas uma mão, pode ser adicionada uma segunda mão com CM e MOV simétricos, tal qual ilustra a Figura 2.

Figura 2 - Simetria de duas mãos no sinal de FACA em Libras.

\begin{tabular}{|l|l|l|l|}
\hline GLOSA & ICON. & OATES & INES \\
\hline Est. 2 & FACA & & \\
\hline & & & \\
& & & \\
& FACA & \\
\hline
\end{tabular}

Fonte: DINIZ (2010, p. 88).

Na Figura 2, Diniz (2010) apresenta a mudança que ocorre na CM da mão passiva de forma simétrica com a ativa na realização do sinal referente a FACA em Libras.

(b) Deslocamento locativo ocorre por conta da acuidade visual, fazendo com que o sinal mude de locação. Dois tipos de deslocamentos podem ocorrer: acima do pescoço e abaixo do pescoço. Observe-se a Figura 3.

\footnotetext{
${ }^{2}$ Mão não dominante ou passiva é a que serve de sustentação para a realização dos movimentos e de locação para a mão dominante.

${ }^{3}$ Mão dominante ou ativa é a que realiza os movimentos e geralmente são sustentadas pela mão passiva.
}

Revista Moara, n. 55, jan-jul 2020 ISSN: 0104-0944

Recebido em 25/03/2020

Avaliado em 25/06/2020 
Figura 3 - Deslocamento locativo no sinal de GUARDANAPO em Libras.

\begin{tabular}{|l|c|c|c|}
\hline \multicolumn{1}{|c|}{ GLOSA } & ICON. & OATES & INES \\
\hline Est. 2 & & \\
\hline GUARDA- & & \\
NAPO & & \\
& &
\end{tabular}

Fonte: DINIZ (2010, p. 88).

Na Figura 3, tem-se a ocorrência de deslocamento locativo no sinal de GUARDANAPO em Libras, em que ocorre o deslocamento da locação boca para a locação bochecha.

(c) Conteúdo lexical para as mãos ocorre quando um sinal que tem uma característica icônica representada corporalmente passa a transportar essa representação para as mãos, podendo perder seu caráter icônico, tornando-se mais arbitrário, como evidencia a Figura 4.

Figura 4 - Transferência do conteúdo lexical do sinal de CADEIRA em Libras para as mãos.

\begin{tabular}{|l|c|c|c|}
\hline \multicolumn{1}{|c|}{ GLOSA } & ICON. & OATES & INES \\
\hline Est. 5 & & \\
\hline CADEIRA & &
\end{tabular}

Fonte: Diniz (2010, p. 91).

Observa-se a partir da Figura 4, com o sinal de CADEIRA em Libras, representando em um primeiro momento com o ato de sentar-se na cadeira e, com o tempo, esse sinal teve seu sentido lexical transferido para as mãos. 
(d) Assimilação é evidenciada em sinais compostos que se transformam em unitários por meio da assimilação e flexibilidade. Diniz (2010) exemplifica com o sinal de ÁGUA em Libras, que, da realização de um sinal composto passou a ser realizado com apenas um sinal.

Figura 5 - Assimilação do sinal de ÁGUA em Libras.

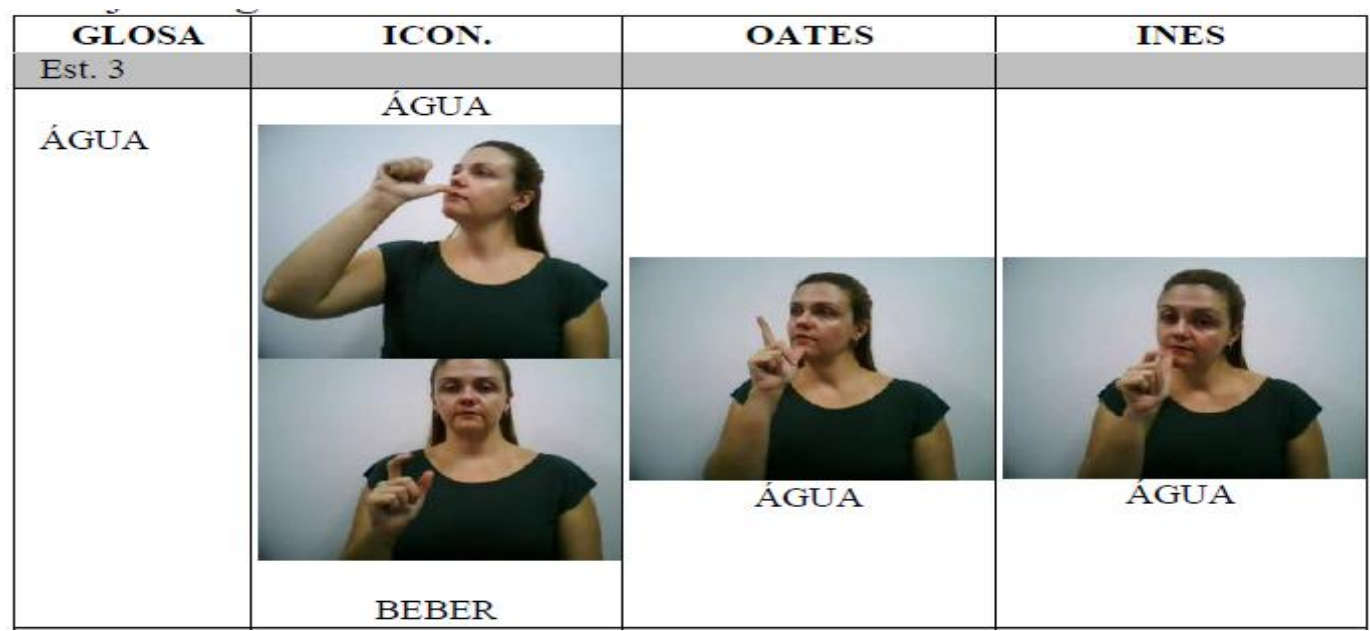

Fonte: Diniz (2010, p. 92).

(e) Deletação de uma mão acontece em sinais realizados com duas mãos, em que uma delas é deletada. Como demonstrado na Figura 6, em que a mão ativa do sinal de LEITE em Libras é deletada, permanecendo, atualmente, somente uma mão.

Figura 6 - Deletação de uma mão no sinal de LEITE em Libras.

\begin{tabular}{|l|c|c|c|}
\hline \multicolumn{1}{|c|}{ GLOSA } & ICON. & OATES & INES \\
\hline Est. 3 & & \\
\hline LEITE & & \\
& & & \\
& LEITE & LEITE & LEITE \\
\hline
\end{tabular}

Fonte: Diniz (2010, p. 94). 
As tendências apresentadas, mesmo que sinteticamente, ilustram parte do processo fonológico a que tais estruturas estão passíveis.

\section{Metodologia}

Para a realização desta pesquisa foram escolhidos três dicionários, um de cada língua de sinais e partir deles selecionamos os sinais que demonstraram ter alguma semelhança paramétrica em sua constituição.

Os dicionários escolhidos foram: Iconographie des signes da LSF, Dicionário da Língua de Sinais do Brasil e HandSpeak ${ }^{4}$. Os dois primeiros dicionários são em formato impresso e o segundo em formato digital.

Desses materiais, selecionamos 21 sinais, sendo 7 sinais de cada língua, que foram organizados em tabelas comparativas. Após, iniciamos a análise identificando as mudanças fonológicas quanto aos parâmetros CM, MOV, LOC e OP ocorridas da LSF para a ASL e para a Libras, em seguida, foram discutidas as tendências gerais de mudanças.

\section{Resultados}

Nesta seção apresentamos o quadro comparativo dos sinais Colher e Garfo nas três línguas analisadas a partir de cada um dos dicionários referenciados na Metodologia. Para efeito de organização, segue-se a ordem LSF, ASL e Libras, respectivamente.

\footnotetext{
${ }^{4}$ Disponível em: <https://www.handspeak.com/>

Revista Moara, n. 55, jan-jul 2020 ISSN: 0104-0944

Recebido em 25/03/2020

Avaliado em 25/06/2020
} 
Quadro 1 - Análise comparativa dos sinais de COLHER e GARFO.

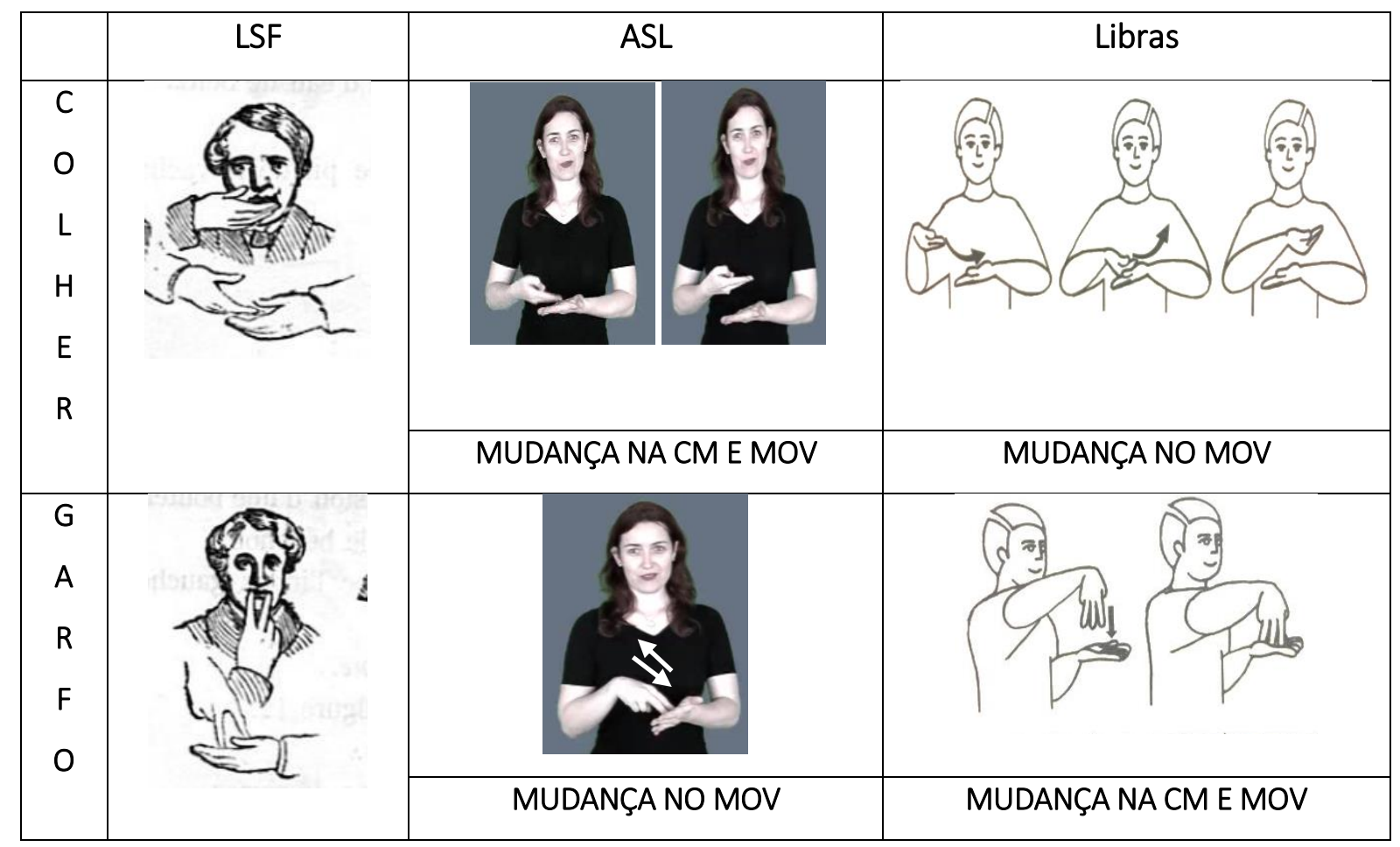

Fonte: Elaboração dos autores, 2020.

Os dados mostrados no Quadro 1 evidenciam as mudanças fonológicas que os sinais podem sofrer em sua configuração paramétrica, e mostram a tendência para a assimilação, uma vez que os sinais compostos realizados com uma longa extensão do movimento ocupando duas locações (no exemplo COLHER na ASL: palma da mão e boca) tendem a sofrer um encurtamento no seu movimento passando a ocupar apenas uma locação. Dito de outra forma, sinais compostos que possuem movimentos que iniciam em uma locação e terminam em outra locação, a LOC1 tende a assimilar a LOC2, mudando para sinais com apenas uma locação. Assumem, portanto, a configuração:

\section{MOVloc1 MOVloc2 > MOVloc1}

Revista Moara, n. 55, jan-jul 2020 ISSN: 0104-0944 
No Quadro 2, mais um exemplo comparativo de como ocorre o processo de mudança para os itens lexicais Carta e Duro.

Quadro 2 - Análise comparativa dos sinais de CARTA e DURO.

ISF

Fonte: Elaboração dos autores, 2020.

Podemos perceber a partir do Quadro 2 que, os dois sinais da ASL mudaram a CM da mão passiva, nesse caso, a mão passiva copiou a CM da mão ativa. Na Libras, as mudanças não ocorreram ou foram mínimas, como no sinal de CARTA e DURO, respectivamente. Dessa forma, entendemos que a mão passiva (MP) dos sinais bimanuais, sinais realizados com duas mãos (XAVIER, 2016), tendem a copiar simetricamente as características paramétricas da mão ativa. A relação observada encontra-se expressa abaixo:

\section{MAcm1 MPcm2 > MAcm1 MPcm1}

No Quadro 3 está exposta a análise comparativa para os verbos Mentir e Saber em cada uma das línguas analisadas. 


\section{Quadro 3 - Análise comparativa dos sinais de MENTIR e SABER}

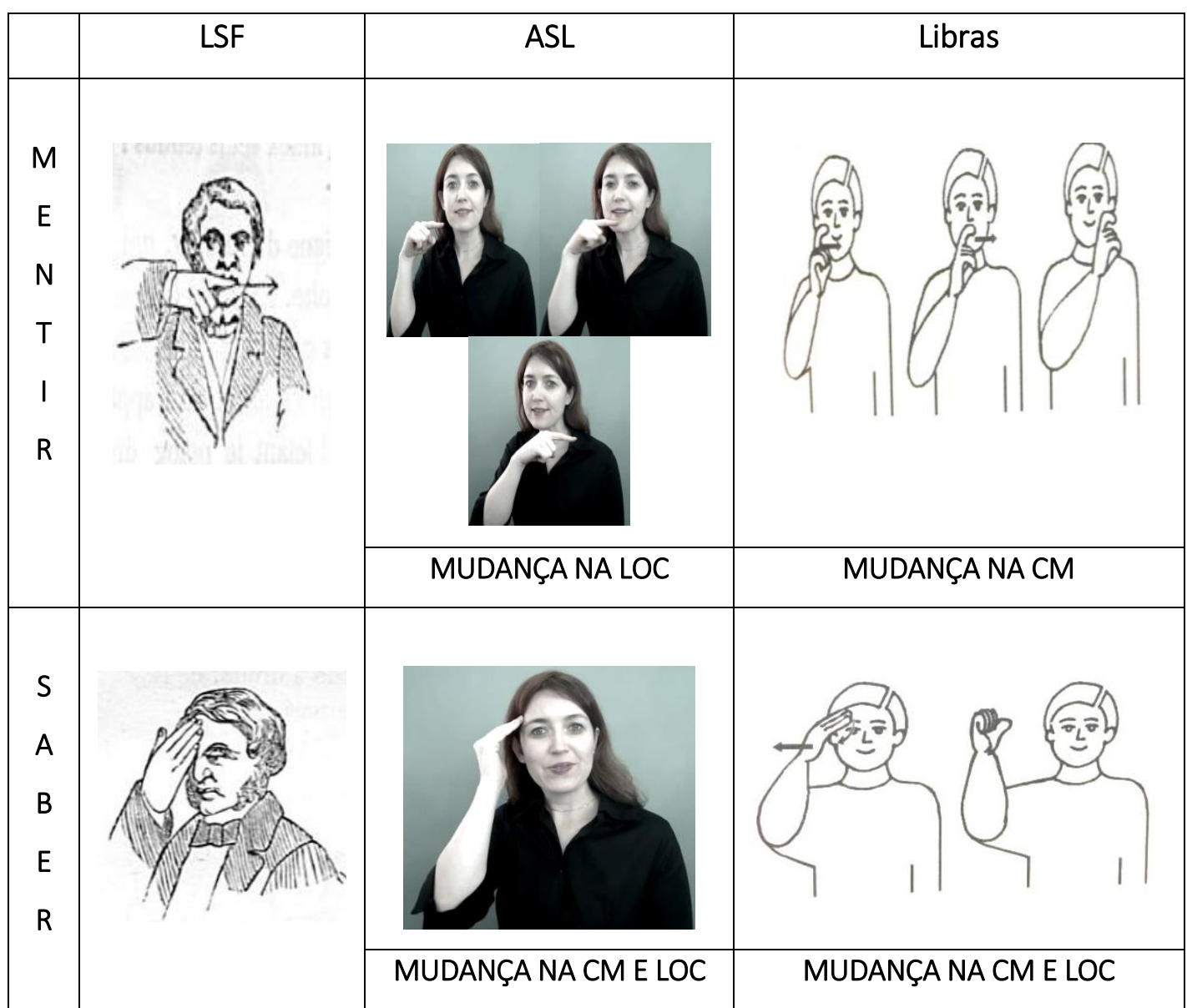

Fonte: Elaboração dos autores, 2020.

A partir das mudanças fonológicas apresentadas no Quadro 3, observa-se a tendência de deslocamento locativo, as mudanças no parâmetro locação ocorrem por conta da acuidade visual, os sinais de MENTIR e SABER na LSF cobriam, de alguma forma, parte do rosto. Ao entrarem em uso nas línguas-filhas, são deslocadas para facilitar a percepção facial. O único sinal que permaneceu, mudando apenas sua $\mathrm{CM}$, foi o sinal de MENTIR na Libras. A ordenação dessa relação está disposta em:

\section{LOCfaceinterna > LOCfaceexterna}

O Quadro 4, ilustra a ocorrência, nas três línguas, para o item Ano.

Quadro 4 - Análise comparativa do sinal de ANO.

\begin{tabular}{|l|l|l|l|}
\hline & LSF & ASL & Libras \\
\hline
\end{tabular}




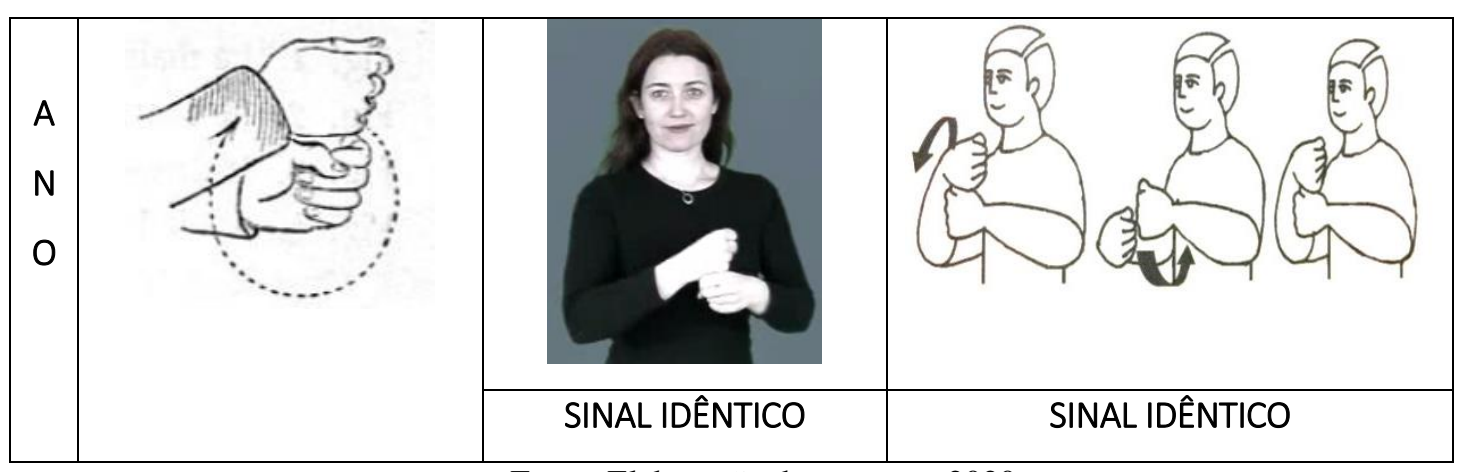

Fonte: Elaboração dos autores, 2020.

No Quadro 4, analisamos que o sinais permaneceram com as mesmas configurações paramétricas $(\mathrm{CP})$ da língua-mãe, dessa maneira, isso evidencia que os sinais ao entrarem em uso nas línguas-filhas podem permanecer com sua estrutura primeira, advinda da língua-mãe. Sendo assim, como tendência, temos:

\section{$\mathrm{CP} 1>\mathrm{CP} 1$}

Sinteticamente, as relações encontradas apresentam os padrões dispostos no Quadro 5:

Quadro 5 - Síntese dos resultados encontrados

\begin{tabular}{|c|c|}
\hline \multicolumn{2}{|c|}{ Configuração / Relação } \\
\hline Configuração 1 & MOVloc1 MOVloc2 > MOVloc1 \\
\hline Configuração 2 & MAcm1 MPcm2 > MAcm1 MPcm1 \\
\hline Configuração 3 & LOCfaceinterna > LOCfaceexterna \\
\hline Configuração 4 & CP1 > CP1 \\
\hline
\end{tabular}

Fonte: Elaboração dos autores, 2020. 


\section{Considerações finais}

As línguas de sinais, assim como as línguas orais, também apresentam um histórico de relações linguísticas. A partir do contexto histórico podemos evidenciar essas relações e analisar os sinais e suas mudanças, criando, dessa forma, tendências gerais de mudanças a partir da configuração paramétrica.

Foram identificadas três tendências gerais de mudança. A primeira, ocorre nos sinais compostos que apresentam duas locações, em que a primeira locação tende a assimilar a segunda passando a constituir apenas uma locação. A segunda, relacionada a sinais bimanuais, observa-se que a mão passiva tende a copiar a CM da mão ativa. A terceira mudança acontece em sinais que interferem a acuidade visual da face e tendem a serem deslocados para os locais externos do rosto. Há ainda, a evidência de um sinal que preservou suas características paramétricas.

Quanto à terceira mudança, percebemos que os sinais da ASL demonstraram uma tendência forte para deslocar os sinais que apresentavam locação em alguma parte do rosto e que de alguma forma interferiam a acuidade visual (COLHER, GARFO, CARTA e MENTIR), na Libras, isso ocorreu apenas em dois sinais (GARFO e SABER). Com os dados apresentados, comparando as duas línguas-filhas, percebemos que os sinais da ASL sofreram mudanças mais consideráveis do que na Libras.

\section{REFERÊNCIAS}

CAPOVILlA, F. C. [et al.] (2017). Dicionário da Língua de Sinais do Brasil: A Libras em suas Mãos. São Paulo: Editora da Universidade de São Paulo.

Dicionário da Língua de Sinais Americana/ASL. Disponível em: <http://www.handspeak.com>. Acesso em: 25 maio 2017.

DINIZ, H. G. A história da Língua de Sinais Brasileira (Libras): um estudo descritivo de mudanças fonológicas e lexicais. Dissertação de Mestrado. Florianópolis, SC, 2010. 
GABAS JR, N. Linguística Histórica. In: MUSSALIN, F. BENTES, A. C. (orgs.). Introdução à Linguística: domínios e fronteiras. Volume $1.9^{\circ}$ ed. rev. São Paulo: Cortez, 2012.

PAIXÃO DE SOUSA, M. C. Linguística Histórica. In: Claudia Catellanos Pfeiffer \& José Horta Nunes (orgs.). Introdução às ciências da linguagem - Linguagem, história e conhecimento. Campinas: Pontes, 2006.

PÉLISSIER, P. (1856). Iconographie des signes faisant partie de "I'Enseignement primaire des sourds-muets". Bibliothèque nationale de France. Disponível em: <gallica.bnf.fr> acesso em: 20 maio 2017.

QUADROS, R.; KARNOPP, L. Língua de Sinais Brasileira: Estudos linguísticos. Porto Alegre: Artmed, 2004.

ROCHA, S. M. O INES e a Educação de Surdos no Brasil. Vol. 01, $2^{\circ}$ edição (DEZ/2008). Rio de Janeiro: INES, 2008.

XAVIER, N. X. A variação na produção de sinais da Libras à luz da Fonologia Gestual. Gradus Revista Brasileira de Fonologia de Laboratório. Vol. 1. $\mathrm{N}^{\mathrm{o}} 1$ (DEZ/2016). Curitiba - PR, 2016. 